\title{
A modified stochastic volatility model based on Gamma Ornstein-Uhlenbeck process and option pricing
}

\author{
Yanhui Mi \\ Department of Statistics, Purdue University \\ 250 N, University Street, West Lafayette IN, 47907 USA
}

Received: 8 May 2016; Accepted: 4 June 2016

Published: 10 August 2016

\begin{abstract}
Stochastic volatility model of the Gamma Ornstein-Uhlenbeck possess authentic capability of both capturing some stylized features of financial time series and pricing European options. In this work we modify the Gamma OU model from the viewpoint of Monte Carlo simulation, which is crucial in both model inference and exotic option pricing. We discuss topics related to the measure transformation between objective and risk-neutral measures, arbitrage-free and market incompleteness of the new model. Furthermore, we investigate the performance of this model in European options pricing and an empirical application is presented.
\end{abstract}

Keywords: Gamma Ornstein-Uhlenbeck processes; gamma-beta relationship; objective and risk-neutral measures; incomplete markets; option pricing; Monte Carlo.

\section{Introduction}

The standard models for portfolio allocation (Merton, 1973) and for option pricing (Black and Scholes, 1973) both assume that continuously compounded returns are normally distributed. The central limit theorem is often invoked as a primary motivation for this assumption. If returns are realized as the sum of a large number of independent influences, then one can anticipate that returns will in fact be normally distributed.

This is an Open Access article published by World Scientific Publishing Company. It is distributed under the terms of the Creative Commons Attribution 4.0 (CC-BY) License. Further distribution of this work is permitted, provided the original work is properly cited. 
Unfortunately, it is well documented that the assumed normality of the return distribution is violated in both the time-series data and in option prices. It is widely accepted that financial time series of different assets share a common set of wellestablished stylized features. For example, log-returns are known to display heavy tailed and high kurtosis distributions. In financial derivative market, it has been clear that the standard option pricing model of Black and Scholes has been inconsistent with options data for at least a decade. The model in fact implies that the informational content of the option surface is one-dimensional. If the model were to be empirically relevant, then one could infer the prices of options at all strikes and maturities from the price of any single option. This property calls into question the associated activity of price under the Black and Scholes model.

On the theoretical side, arguments have been proposed by Geman et al. (2001) which suggest that price processes for financial assetsmust have a jump component but they need not have a diffusion component. Their argument rests on recognizing that all price processes of interest may be regarded as Brownian motion subordinated to a random clock. An example of infinite activity pure jump Levy processes is variance gamma (VG) model studied by Madan and Seneta (1990) and its asymmetric extension studied by Madan et al. (1998). The main feature missing from the Levy model described above is the fact that volatility is changing stochastically over time and negatively correlated with the stock price. It has been observed that the estimated volatilities change stochastically over time and are clustered. In derivative market, the pure jump Levy processes can not perform well when one considers the variation of option prices across maturity (Konikov and Madan, 2002).

These empirical facts in both the time-series data and in option prices are successfully captured by a new class of models in which the stochastic variance of log-returns is constructed via a mean-reverting stationary process of the OrnsteinUhlenbeck (OU) type driven by a subordinator. These stochastic volatility models have been introduced and thoroughly studied by Barndorff-Nielsen and Shephard (2001) and will be, hereafter, termed BNS models.

Traditional inference approaches for BNS model to empirical financial timeseries data are Bayesian inference based on Markov Chain Monte Carlo (MCMC) algorithm which involve the posterior distributions of all parameters given some priors. Due to the evolution of dynamics of volatility in BNS model is complicate, the simulation for the posterior distribution of each parameter is computationally burdensome (Barndorff-Nielsen and Shephard, 2001).

Again, the path-dependent exotic options have become popular in the OTC market in the last two decades. Examples of these exotic options are lookback options, barrier options and asian options. The prices of barrier and lookback options under Black-Scholes model have closed formular. But the asian option 
does not have closed formular even in Black-Scholes model. If we use Levy model or more advanced stochastic volatility models to price exotic options, the closed formulars do not exist. Besides, if the payoff of option is dependent of several underlying variables, the closed pricing formular can not be derived generally. In both case, we need to make use of numerical procedure such as Monte Carlo techniques. The Monte Carlo method is based on the simulation of the stock-price process. Barndorff-Nielsen and Shephard (2001) showed that the simulation of BNS model needs series representation. The procedure in this simulation involves approximation to the stochastic intergral and may introduce large variance and reduce efficiency in pricing path-dependent exotic options.

In this paper we first focus on the measure transformation and option pricing of BNS model with Gamma OU process. And then we proposed a modified stochastic volatility model SVBGAR based on BNS model. The new model has same properties as BNS model such that the financial returns display fatter tailed distribution and volatility clustering effect. Moreover, we investigate the option pricing framework of SVBGAR model. Typically, financial markets where investors can only trade in a riskless asset and in an underlying stock with stochastic volatility are incomplete, and in SVBGAR model is no exception. In this work we derived measure transformation between the objective measure and the equivalent martingale measure (or risk-neutral measure). After that, the performance of SVBGAR model in pricing European option was investigated.

The paper proceeds as follows. Section 2 reviews the variance gamma model which gives the foundation of SVBGAR model in terms of the returns distribution. Section 3 goes through the measure transformation and option pricing techniques of BNS model. Section 4 presents the SVBGAR model and its performance in option pricing. Section 5 concludes with a few remarks.

\section{Variance Gamma Model in Finance}

The variance gamma (VG) Levy processes were proposed in Carr et al. (1998) to model the $\log$ return process $X_{t}:=\log \left(S_{t} / S_{0}\right)$ of a financial asset. The VG model can be seen as a Brownian motion with drift time-deformed by a random clock. That is, $X_{t}$ enjoys the representation

$$
X_{t}=b t+\theta \tau(t)+\sigma W(\tau(t)),
$$

where $\sigma>0, \theta \in R$ are given constants, $W$ is Wiener process, and $\tau(t)$ is a gamma Levy subordinator (nondecreasing Levy process), which has gamma distributed with scale parameter $\beta:=\kappa$ and shape parameter $\alpha:=t / \kappa$. A well known fact is that Levy process has independent and stationary increments. By the definition 
of variance gamma process, the increments $X_{t+\delta}-X_{t}$ has Variance gamma distribution, which is gamma variance-mean mixture of normal distribution as: if $\left(X_{\delta} \mid \tau_{\delta}\right) \sim N\left(b \delta+\theta \tau_{\delta}, \sigma^{2} \tau_{\delta}\right)$ and $\tau_{\delta} \sim \operatorname{Gamma}(\delta / \kappa, \kappa)$, then $X_{\delta}$ has same distribution as any increments in $X_{t+\delta}-X_{t}$ of variance gamma process.

Variance gamma model has been researched in different financial literatures. The empirical property of variance gamma model such that it has fatter tail and higher kurtosis than Brownian motion was investigated in Madan et al. (1990). The option pricing framework was given in Carr et al. (1998). But VG model does not characterize the dependence of the financial returns and volatility clustering effect which are observed in historical time series data.

\section{Non-Gaussian Ornstein-Uhlenbeck-Based Stochastical Volatility Model}

One way to incorporate both variance gamma distribution and stochastical volatility effect is using non-Gaussian Ornstein-Uhlenbeck-based stochastical volatility model (Barndorff-Nielsen and Shephard, 2001). The key points of this approach are the presence of jumps in the volatility, flexibility in the choice of marginal distributions for volatility and the availability of closed form expression for characteristic functions of integrated volatility and returns. In this model, the stock process $S_{t}$ under objective measure is given by

$$
\begin{gathered}
S_{t}=S_{0} e^{X_{t}}, \\
d X_{t}=\left(\mu+\beta \sigma_{t}^{2}\right) d t+\sigma_{t} d W_{t}+\rho d z_{t}, \\
d \sigma_{t}^{2}=-\lambda \sigma_{t}^{2} d t+d z_{t}, \quad \sigma_{0}^{2}>0,
\end{gathered}
$$

where $\mu, \beta, \rho$ and $\lambda$ are real constants with $\lambda>0$ and $\rho<0, W_{t}$ is a standard Brownian motion and $z_{t}$ is the background driving Levy process, a subordinator without drift. The term $\beta \sigma^{2}$ in Eq. (3) corresponds to the volatility risk premium and the last term $\rho d z_{t}$ accounts for the leverage effect. In the sequel we will call such model BNS model.

In the BNS model (3), the volatility at increases by jumps and decays exponentially between two jumps. When $\rho \neq 0$, each jump in the volatility is associated to a jump in the price process, whose size is proportional to the size of volatility jump. The jumps can be interpreted as the arrival new information on the market, triggering an increase in volatility and a simultaneous fall of asset price. When $\rho=0$, the volatility still moves by jumps but the price process has continuous sample paths.

Non-Gaussian Ornstein-Uhlenbeck processes in (4) are closely linked to a class of infinitely divisible distributions called self-decomposable distributions. 
In essence, the process $\sigma_{t}^{2}$ is strictly stationary on the positive half-line, i.e. there exists a law $D$, called the stationary law or the marginal law, such that $\sigma_{t}^{2}$ will follow the law $D$ for every $t$ if the initial $\sigma_{0}^{2}$ is chosen according to $D$. The process $\sigma_{t}^{2}$ moves up entirely by jumps and then tails off exponentially. The gamma distribution is self-decomposable. In this respect it is interesting to consider an OU process which admits a gamma distribution as its stationary measure and compare the corresponding stochastic volatility models based on the two processes. In particular, this will allow us to see, whether, in addition to marginal properties of at, jumps in the volatility are important. Suppose that the $\sigma_{t}^{2}$ has stationary gamma distribution with shape $c$ and rate $\alpha$, which has density $f_{\text {Gamma }}(x)=\frac{\alpha^{c}}{\Gamma(c)} x^{c-1} e^{-\alpha x}$ for $x>0$. Barndorff-Nielsen and Shephard (2001) showed that the $z_{t}$ is a compound poisson variable $\sum_{j=1}^{N_{t}} G_{j}$, where $N_{t}$ is a Poisson process with intensity parameter $\lambda c$, i.e. $E\left[N_{t}\right]=\lambda c$ and $\left\{G_{j}, j=1,2, \ldots\right\}$ is an independent and identically distributed sequence; each $G_{j}$ follows a $\operatorname{Gamma}(1, \alpha)$ law. Since the $z_{t}$ is compound Poisson, it only jumps a finite number of times in every compact interval. Hence, the process $\sigma_{t}^{2}$ also jumps a finite number of times in every compact (time) interval.

\subsection{BNS model under risk-neutral measure}

Recall that a probability measure $Q$ is called an risk-neutral measure if it is equivalent to the objective measure and if under $Q$ the discounted price process $e^{-r t} S_{t}$ is a martingale. Under one of such measure the models (2), (3) and (4) has the form

$$
\begin{gathered}
S_{t}=S_{0} e^{X_{t}}, \\
d X_{t}=\left(r-l(\rho)-\frac{1}{2} \sigma_{t}^{2}\right) d t+\sigma_{t} d W_{t}^{Q}+\rho d z_{t}^{Q}, \\
d \sigma_{t}^{2}=-\lambda \sigma_{t}^{2} d t+d z_{t}^{Q}, \quad \sigma_{0}^{2}>0,
\end{gathered}
$$

where $r$ is the intereste rate and the superscript $Q$ refers to the fact that $W_{t}$ and $Z_{t}$ are, respectively, standard Brownian motion and subordinator with Laplace exponent $l(u)=\log \left(E\left[e^{u z_{1}}\right]\right)$. In the case of that $\sigma_{t}^{2} \sim \operatorname{Gamma}(\tilde{c}, \tilde{\alpha})$ under the risk-neutral probability, $z_{t}^{Q}=\sum_{j=1}^{N_{t}} G_{j}$, where where $N_{t}$ is a Poisson process with intensity parameter $\tilde{\lambda} \tilde{c}$, and $\left\{G_{j}, j=1,2, \ldots\right\}$ is an independent and identically distributed $\operatorname{Gamma}(1, \tilde{\alpha})$ variables. The characteristic function of $z_{t}$ is by simple calculation

$$
\begin{aligned}
E\left[e^{i u z_{t}}\right] & =E\left[e^{i u \sum_{j=1}^{N_{t}} G_{j}}\right]=\exp \left\{\tilde{\lambda} \tilde{c} t \int_{z>0}\left(e^{i u z}-1\right) f(z) d z\right\} \\
& =\exp \left\{t\left(\tilde{\lambda} \tilde{c} \int_{z>0}\left(e^{i u z}-1\right) f(z) d z\right)\right\}=\exp \left\{t\left(\int_{z>0}\left(e^{i u z}-1\right) \tilde{v}(d z)\right)\right\},
\end{aligned}
$$


since the Levy measure of $z_{t}$ has a density $\tilde{v}(d z)=\tilde{v}(z) d z=\tilde{\lambda} \tilde{c} f(z) d z$. Similarly the moment generating function of $z_{t}$ is

$$
E\left[e^{u z_{t}}\right]=\exp \left\{t\left(\int_{z>0}\left(e^{u z}-1\right) \tilde{v}(d z)\right)\right\} .
$$

Then

$$
\tilde{l}(u)=\log E\left[e^{u z_{1}}\right]=\int_{z>0}\left(e^{u z}-1\right) \tilde{v}(d z)=\frac{u \tilde{\lambda} \tilde{c}}{\tilde{\alpha}-u} .
$$

To see why (5)-(7) hold under the risk-neutral measure. Let $x_{t}=-r t+X_{t}$, consider $Y_{t}=e^{-r t+X_{t}}=e^{x_{t}}$, where $x_{t}=\int_{0}^{t}\left(-l(\rho)-\frac{1}{2} \sigma_{t}^{2}\right) d s+\int_{0}^{t} \sigma_{s} d W_{s}^{Q}+\rho z_{t}^{Q}$ $=\int_{0}^{t}\left(-l(\rho)-\frac{1}{2} \sigma_{t}^{2}\right) d s+\int_{0}^{t} \sigma_{s} d W_{s}^{Q}+\rho \sum_{j=1}^{N_{t}} G_{j}$, and the $x_{t}$ 's continue part $\hat{X}_{t}^{c}=$ $\int_{0}^{t}\left(-l(\rho)-\frac{1}{2} \sigma_{t}^{2}\right) d s+\int_{0}^{t} \sigma_{s} d W_{s}^{Q}$. Apply Ito's formula for Levy process (Cont 2004, Theorem 8.14) with respect function of $f\left(x_{t}\right)=e^{x_{t}}$,

$$
\begin{aligned}
Y_{t}-Y_{0}= & \int_{0}^{t} e^{x_{s}} d x_{s}^{c}+\frac{1}{2} e^{x_{s}} d x_{s}^{c} d x_{s}^{c}+\sum_{s \leq t}\left(e^{x_{s}}-e^{x_{s-}}\right) \\
= & \int_{0}^{t} e^{x_{s}}\left(-l(\rho)-\frac{1}{2} \sigma_{s}^{2}\right) d s+\int_{0}^{t} e^{x_{s}} \sigma_{s} d W_{s}^{Q} \\
& +\frac{1}{2} \int_{0}^{t} e^{x_{s}} \sigma_{s}^{2} d s+\sum_{s \leq t}\left(e^{x_{s}}-e^{x_{s-}}\right) \\
= & \int_{0}^{t} e^{x_{s}}(-l(\rho)) d s+\int_{0}^{t} e^{x_{s}} \sigma_{s} d W_{s}^{Q}+\sum_{s \leq t} e^{x_{s-}}\left(e^{\Delta x_{s}}-1\right) \\
= & \int_{0}^{t} e^{x_{s}}(-l(\rho)) d s+\int_{0}^{t} e^{x_{s}} \sigma_{s} d W_{s}^{Q}+\sum_{s \leq t} e^{x_{s-}}\left(e^{\rho \Delta z_{s}}-1\right) \\
= & \int_{0}^{t} e^{x_{s}}(-l(\rho)) d s+\int_{0}^{t} e^{x_{s}} \sigma_{s} d W_{s}^{Q}+\int_{0}^{t} \int_{z>0} e^{x_{s-}}\left(e^{\rho z}-1\right) J(d z, d s) \\
= & \int_{0}^{t} e^{x_{s}}(-l(\rho)) d s+\int_{0}^{t} e^{x_{s}} \sigma_{s} d W_{s}^{Q} \\
& +\int_{0}^{t} \int_{z>0} e^{x_{s-}}\left(e^{\rho z}-1\right)(J(d z, d s)-\tilde{v}(d z) d s) \\
& +\int_{0}^{t} \int_{z>0} e^{x_{s}}\left(e^{\rho z}-1\right) \tilde{v}(d z) d s \\
= & \int_{0}^{t} e^{x_{s}}\left(\int_{z>0}\left(e^{\rho z}-1\right) \tilde{v}(d z)-l(\rho)\right) d s+\int_{0}^{t} e^{x_{s}} \sigma_{s} d W_{s}^{Q} \\
& +\int_{0}^{t} \int_{z>0} e^{x_{s-}}\left(e^{\rho z}-1\right)(J(d z, d s)-\tilde{v}(d z) d s) \\
= & \int_{0}^{t} e^{x_{s}} \sigma_{s} d W_{s}^{Q}+\int_{0}^{t} \int_{z>0} e^{x_{s-}}\left(e^{\rho z}-1\right)(J(d z, d s)-\tilde{v}(d z) d s) \\
&
\end{aligned}
$$


where $J(d z, d x)$ is the Jump random measure of $z_{t}$ and the two terms in the last equation are martingales (Cont 2004, Theorem 8.7). Then $Y_{t}$ is a martingale and $e^{-r t} S_{t}=S_{0} Y_{t}$ is also a martingale.

Next we can check if the risk-neutral measure exists for this model. Under object measure, $Y_{t}=e^{-r t} S_{t} / S_{0}=\exp \left\{\int_{0}^{t}\left(\mu-r+\beta \sigma_{s}^{2}\right) d s+\int_{0}^{t} \sigma_{s} d W_{s}+\rho z_{t}\right\}$. Apply Ito's lemma to the $Y_{t}$, we have

$$
\begin{aligned}
Y_{t}-Y_{0}= & \int_{0}^{t} Y_{s}\left(\mu-r+\left(\frac{1}{2}+\beta\right) \sigma_{s}^{2}\right) d s \\
& +\int_{0}^{t} Y_{s-}\left(e^{\rho z}-1\right) J(d s, d z)+\int_{0}^{t} Y_{s} \sigma_{s} d W_{s} .
\end{aligned}
$$

Define $\theta_{s}=\frac{\mu-r+\left(\frac{1}{2}+\beta\right) \sigma_{s}^{2}+\tilde{l}(\rho)}{\sigma_{s}}=\frac{\mu-r+\left(\frac{1}{2}+\beta\right) \sigma_{s}^{2}+\frac{\rho \tilde{\alpha} \tilde{c}}{\tilde{\alpha}-\rho}}{\sigma_{s}}$, we have

$$
\mu-r=\theta_{s} \sigma_{s}-\tilde{l}(\rho)=\theta_{s} \sigma_{s}-\int_{z>0}\left(e^{u z}-1\right) \tilde{v}(d z) .
$$

We also define

$$
\begin{aligned}
& Z_{1}(t)=\exp \left\{-\int_{0}^{t} \theta_{s} d W_{s}-\frac{1}{2} \int_{0}^{t} \theta_{s}^{2} d s\right\} \\
& Z_{2}(t)=\exp \left\{(\lambda c-\tilde{\lambda} \tilde{c}) t+\sum_{s \leq t} \log \left(\frac{\tilde{v}(d z)}{v(d z)}\right)\right\}=e^{(\lambda c-\tilde{\lambda} \tilde{c}) t} \prod_{i=1}^{N_{t}} \frac{\tilde{\lambda} \tilde{c} G_{i}(1, \tilde{\alpha})}{\lambda c G_{i}(1, \alpha)}, \\
& Z_{3}(t)=Z_{1}(t) Z_{2}(t) .
\end{aligned}
$$

Fix a positive $T$ and define $d Q=Z_{3}(T) d P$, by Girsanov's Theorem (Shreve, 2004, Theorem 11.6.9),

$$
W_{t}^{Q}=W_{t}+\int_{0}^{t} \theta_{s} d s
$$

is a Brownian motion under $Q$, and $z_{t}$ is a compound poisson process with intensity $\tilde{\lambda} \tilde{c}$ and jump distribution of $G_{i}(1, \tilde{\alpha})$. We plug (13) and 14 into (12) and then $Y_{t}$ has the dynamics in (11). So the risk-neutral measure exists for this model. But the solution of $\theta_{s}$ is not unique, then there exsit many different risk-neutral measure and the market under this model is not complete.

\subsection{Option pricing under BNS model}

In this section we assume the model is under risk-neutral measure. One way to price the European options given the Characteristic function of the $\log S_{t}$ is using Fourier transform (Carr et al., 1999). 
As stated in Barndorff-Nielsen et al. (2002) and Carr et al. (2003), the Characteristic function of the $\log S_{t}$ under models (5)-(7) is given by

$$
\begin{aligned}
\phi(u)=E\left[e^{i u \log S_{t}}\right]= & \exp \left\{i u \log S_{0}+i u t\left(r-\frac{\lambda c \rho}{\alpha-\rho}\right)-\sigma_{0}^{2} \frac{\left(1-e^{-\lambda t}\right)}{\lambda} \frac{\left(u^{2}+i u\right)}{2}\right. \\
& +\frac{\alpha \lambda c}{\lambda(\alpha-i u \rho)+\frac{\left(u^{2}+i u\right)}{2}} \log \left(1+\frac{\left(u^{2}+i u\right)}{2} \frac{1}{\alpha-i u \rho} \frac{\left(1-e^{-\lambda t}\right)}{\lambda}\right) \\
& \left.+\lambda c t \frac{2 i \lambda u \rho-u^{2}+i u}{2 \lambda(\alpha-i u \rho)+u^{2}+i u}\right\} .
\end{aligned}
$$

Let $\alpha_{0}$ be a positive constant such that the $\alpha$ th moment of the stock exists. Carr et al. (1998) showed that the price a European call option with strike $K$ at the time of maturity $T$ is

$$
C(K, T)=\frac{e^{-\alpha_{0} K}}{\pi} \int_{0}^{\infty} e^{-i v} K \Psi(v) d v
$$

where

$$
\Psi(v)=\frac{e^{-r T} \phi\left(v-\left(\alpha_{0}+1\right) i\right)}{\alpha_{0}+\alpha_{0}-v^{2}+i(2 \alpha+1) v} .
$$

The fast Fourier transform can be used to invert the generalized Fourier transform of the call price. Put options can be priced using the put-call parity. BarndorffNielsen et al. (2002) and Carr et al. (2003) calibrated this model and then price European options. The results showed that the option prices of BNS model are strongly consistent with market prices and gave the evdience of the validity of BNS model.

The Fourier transformation method is applied to European options well and efficiently, but it can not be used in American and Exotic options. Another method for pricing options is to use a Monte Carlo simulation. The main advantage of this method is that complex and exotic derivatives can be treated easily which is very important in applications, since little is known about functionals of stochastic volatility models. Moreover, options on several assets can also be handled easily using Monte Carlo simulations. The main drawback of Monte Carlo methods is the slow computational speed. The European call option price is provided by the discounted expected payoff under a risk-neutral measure, i.e.

$$
C(K, T)=e^{-r T} E\left[\left(S_{T}-K\right)^{+}\right] .
$$

The crux of pricing European options with Monte Carlo methods is to simulate the terminal value of asset price $S_{T}$. Let $S_{T_{k}}$ for $k=1, \ldots, N$ denote the simulated values; then, the option price $C(K, T)$ is estimated by the average of the prices for 
the simulated asset values, that is

$$
\hat{C}(K, T)=e^{-r T} \sum_{k=1}^{N}\left(S_{T_{k}}-K\right)^{+},
$$

and by the Law of Large Numbers we have that

$$
\hat{C}(K, T) \rightarrow C(K, T) \text { as } N \rightarrow \infty .
$$

If we want to simulate the stock process, we assume that the log return process $X_{t}=\log \left(S_{t}\right)$ is sampled during a fixed time interval [0;T] at evenly spaced times $t_{i}=i \delta, i=1, \ldots, n$, where $\delta=T / n$.

Since we know $z_{t}=\sum_{j=1}^{N(t)} G_{j}(1, \alpha)=\sum_{j=1}^{N(t)} \operatorname{Exp}_{j}(\alpha)$, the $\sigma_{t}^{2}$ of (7) can be expressed as by Ito's lemma

$$
\begin{aligned}
\sigma_{t}^{2} & =\sigma_{0}^{2} e^{-\lambda t}+e^{-\lambda t} \int_{0}^{t} e^{-\lambda s} d z_{s}=\sigma_{0}^{2} e^{-\lambda t}+e^{-\lambda t} \sum_{s \leq t} e^{\lambda s} \Delta z_{s} \\
& =\sigma_{0}^{2} e^{-\lambda t}+e^{-\lambda t} \sum_{j=1}^{N(t)} e^{\lambda s_{j}} G_{j}(1, \alpha) \\
& =\sigma_{0}^{2} e^{-\lambda t}+e^{-\lambda t} \sum_{j=1}^{N(t)} e^{\lambda s_{j}}\left(-\frac{1}{\alpha} \log \left(u_{j}\right)\right),
\end{aligned}
$$

where $u_{j}$ are i.i.d. uniform variables in $[0,1]$. Then we have

$$
\begin{aligned}
\sigma_{t_{i}}^{2} & =\sigma_{0}^{2} e^{-\lambda t_{i}}+e^{-\lambda t_{i}} \int_{0}^{t_{i}} e^{-\lambda s} d z_{s}, \\
\sigma_{t_{i-1}}^{2} & =\sigma_{0}^{2} e^{-\lambda t_{i-1}}+e^{-\lambda t_{i-1}} \int_{0}^{t_{i-1}} e^{-\lambda s} d z_{s},
\end{aligned}
$$

and

$$
\begin{aligned}
\sigma_{t_{i}}^{2} & =e^{-\lambda \delta} \sigma_{t_{i-1}}^{2}+e^{-\lambda t_{i}} \int_{t_{i-1}}^{t_{i}} e^{\lambda s} d z_{s} \\
& =e^{-\lambda \delta} \sigma_{t_{i-1}}^{2}+e^{-\lambda t_{i}} \sum_{j=N\left(t_{i-1}\right)+1}^{N\left(t_{i}\right)} e^{\lambda s_{j}}\left(-\frac{1}{\alpha} \log \left(u_{j}\right)\right) .
\end{aligned}
$$

Given the $\sigma_{t_{i}}^{2}$, we have an approximate discrete version of (5):

$$
\begin{aligned}
\log \left(S_{t_{i}}\right)-\log \left(S_{t_{i-1}}\right) & =\left(r-l(\rho)-\frac{1}{2} \sigma_{t_{i}}^{2}\right) \delta+\sigma_{t_{i}} \sqrt{\delta} \epsilon_{i}+\rho \sum_{t_{i \rightarrow 1} \leq s \leq t_{i}} \Delta z_{s} \\
& =\left(r-l(\rho)-\frac{1}{2} \sigma_{t_{i}}^{2}\right) \delta+\sigma_{t_{i}} \sqrt{\delta} \epsilon_{i}+\rho \sum_{j=N\left(t_{i-1}\right)+1}^{N\left(t_{i}\right)} G_{j}(1, \alpha),
\end{aligned}
$$


where $\epsilon_{i}$ are i.i.d $\mathrm{N}(0,1)$. From (19) and (18), the log-returns have variance gamma distribution when $\rho=0$. Also, this model has volatility clustering effect due to the stochastic volatility. We can use this model to simulate final price $S_{T}$ to price European options and the path of $\left(S_{t}\right)_{0 \leq t \leq T}$ to price path-dependent exotic options.

\section{A Modified Non-Gaussian OU Stochastical Volatility Model: SVBGAR}

The simulation of random term $e^{-\lambda t_{i}} \sum_{j=N\left(t_{i-1}\right)+1}^{N\left(t_{i}\right)} e^{\lambda s_{j}}\left(-\frac{1}{\alpha} \log \left(u_{j}\right)\right)$ in (18) is complex due to stochastic intergral with respect to $z_{t}$ which is a compount poisson process. We use gamma subordinator and gamma-beta algebra to build a simple model such that the increments of log-stocks under the new model still has variance gamma distribution and volatility clustering effect.

We state two lemmas which give the relation between gamma and beta distribution, which is the fundamental principle to propose new model. The proof can be found in Lewis et al. (1989).

Lemma 1. Suppose that $X \sim \operatorname{Gamma}(\alpha, r), Y \sim \operatorname{Gamma}(\beta, r)$, and $X$ and $Y$ are independent. Then

(a) $X+Y \sim \operatorname{Gamma}(\alpha+\beta, r)$, and

(b) $\frac{X}{X+Y}$ follows $\operatorname{Beta}(\alpha, \beta)$ and is independent of $X+Y$.

Lemma 2. If random variables $X$ and $Y$ are independent with $X \sim \operatorname{Beta}(\alpha, \beta)$ and $Y \sim \operatorname{Gamma}(\alpha+\beta, r)$, then $X Y \sim \operatorname{Gamma}(\alpha, r)$.

Let $e_{t}$ is a Gamma process with Levy density $v(d x)=\frac{a e^{-\alpha x}}{x} 1_{x>0} d x$, then the probability density of $e_{t}$ is

$$
f_{e_{t}}(x)=\frac{\alpha^{a t}}{\Gamma(a t)} x^{a t-1} e^{-\alpha x} .
$$

Then the increments of $e_{t}$ at $\left[t_{i-1}, t_{i}\right]$ is $e_{i}=e_{t_{i}}-e_{t_{i-1}}$, which has following distribution as $e_{\delta} \sim \operatorname{Gamma}(a \delta, \alpha)$. We know $\sigma_{t_{i-1}}^{2}$ in (18) follows $\operatorname{Gamma}(c, \alpha)$ distribution and let $b_{i} \sim \operatorname{Beta}(c-a \delta, \delta)$ which is independent with $\sigma_{t_{i-1}}^{2}$ and $e_{i}$, then $b_{i} \sigma_{t_{i-1}}^{2} \sim \operatorname{Gamma}(c-a \delta, \alpha)$ by Lemma 2 . Then we define

$$
\sigma_{t_{i}}^{2}=b_{i} \sigma_{t_{i-1}}^{2}+e_{i} .
$$

By Lemma $1, \sigma_{t_{i}}^{2} \sim$ Gamma $(c, \alpha)$. The $\sigma_{t_{i}}^{2}$ in (18) and (20) have same marginal distribution and auto-regression structure but the simulation in (20) is much simpler. 
In order to consider the leverage effect of stock price, we consider the discrete model for $S_{t}$ as

$$
\begin{aligned}
& \log \left(S_{t_{i}}\right)-\log \left(S_{t_{i-1}}\right) \\
= & \left(r-l(\rho)-\frac{1}{2} \sigma_{t_{i}}^{2}\right) \delta+\sigma_{t_{i}} \sqrt{\delta} \epsilon_{i}+\rho\left(e_{t_{i}}-e_{t_{i-1}}\right),
\end{aligned}
$$

where $\epsilon_{i}$ are i.i.d N(0,1). From (21) and (20), the log-returns still have variance gamma distribution when $\rho=0$. Also, this model has leverage and volatility clustering effect. We call this model as stochastic volatility model with betagamma autoregressive (SVBGAR).

The continuous model corresponding to (21) is

$$
\begin{gathered}
S_{t}=S_{0} e^{X_{t}} \\
d X_{t}=\left(r-l(\rho)-\frac{1}{2} \sigma_{t}^{2}\right) d t+\sigma_{t} d W_{t}+\rho d e_{t} .
\end{gathered}
$$

We can use simliar skills as (11) and show that if

$$
l(\rho)=\log \left(E\left[e^{\rho e_{1}}\right]\right)=-a \log \left(1-\frac{\rho}{\alpha}\right),
$$

then $e^{-r t} S_{t}$ in (22) and (23) is a martingale and the model is risk-neutral. This model is more efficient than BNS model when we price European and exotic options using Monte Carlo simulation.

\subsection{SVBGAR model under objective and risk-neutral measure}

In this section, we use different notation as previous section. Assume the stock process $S_{t}$ under objective measure is given by

$$
\begin{gathered}
S_{t}=S_{0} e^{X_{t}} \\
d X_{t}=\left(\mu+\beta \sigma^{2}\right) d t+\sigma_{t} d W_{t}+\rho d e_{t}, \\
\sigma_{t_{i}}^{2}=b_{i} \sigma_{t_{i-1}}^{2}+e_{i}, \sigma_{0}^{2}>0,
\end{gathered}
$$

where $W_{t}$ is Brownian motion, $e_{t}$ is a gamma process with Levy density $v(d x)=$ $\frac{a e^{-\alpha x}}{x} 1_{x>0} d x$ and $b_{i} \sim \operatorname{Beta}(c-a \delta, \delta)$. We have

$$
e^{-r t} S_{t}=S_{0} e^{Y_{t}}
$$

where

$$
Y_{t}=\int_{0}^{t}\left(\mu-r+\beta \sigma_{s}^{2}\right) d s+\int_{0}^{t} \sigma_{s} d W_{s}+\rho e_{t}
$$


Under risk-neutral measure, the stock process $S_{t}$ is given from (22), (23) and (20):

$$
\begin{gathered}
S_{t}=S_{0} e^{X_{t}}, \\
d X_{t}=\left(r-l(\rho)-\frac{1}{2} \sigma_{t}^{2}\right) d t+\sigma_{t} d W_{t}^{Q}+\rho d e_{t}^{Q}, \\
\sigma_{t_{i}}^{2}=b_{i}^{Q} \sigma_{t_{i-1}}^{2}+e_{i}^{Q}, \quad \sigma_{0}^{2}>0,
\end{gathered}
$$

where $W_{t}^{Q}$ is Brownian motion under risk neutral measure, $e_{t}^{Q}$ is a Gamma process with Levy density $\tilde{v}(d x)=\frac{\tilde{a} e-\tilde{\alpha} x}{x} 1_{x>0} d x$ and $b_{i} \sim \operatorname{Beta}(\tilde{c}-\tilde{a} \delta, \delta)$. We have

$$
e^{-r t} S_{t}=S_{0} e_{t}^{Y},
$$

where

$$
Y_{t}=\int_{0}^{t}\left(-l(\rho)-\frac{1}{2} \sigma_{s}^{2}\right) d s+\int_{0}^{t} \sigma_{s} d W_{s}^{Q}+\rho e_{t}^{Q} .
$$

The main theorem we used to measure transformation is stated as below. The proof can be found in Sato (1999, Theorems 33.1 and 33.2).

Theorem 1. Let $\left(X_{t}, P\right)$ and $\left(X_{t}, P^{\prime}\right)$ be two Levy processes on $R$ with characteristic triplets $\left(\sigma_{t}^{2}, v, \gamma_{t}\right)$ and $\left(\sigma_{t}^{\prime 2}, v^{\prime}, \gamma_{t}^{\prime}\right)$. Then $P$ and $P^{\prime}$ are equivalent for all $t>0$ if and only if all following conditions are satisfied:

(i) $\sigma_{t}=\sigma_{t}^{\prime}$.

(ii) The Levy measure are equivalent with

$$
\int_{-\infty}^{\infty}\left(e^{\phi(x) / 2}-1\right)^{2} v(d x)<\infty
$$

where $\phi(x)=\log \left(\frac{d v^{\prime}}{d v}\right)$. When $P$ and $P^{\prime}$ are equivalent, the Radon-Nikodum derivative is

$$
\frac{d P^{\prime}}{d P}=e^{U_{T}},
$$

with

$$
\begin{aligned}
U_{t}= & \int_{0}^{t} \eta_{s} d X_{s}^{c}-\frac{\int_{0}^{t} \eta_{s}^{2} \sigma_{s}^{2} d s}{2}-\int_{0}^{t} \eta_{s} \gamma_{s} d s \\
& +\lim _{\epsilon \rightarrow 0}\left(\sum_{s \leq t,\left|\Delta X_{s}\right|>\epsilon} \phi\left(\Delta X_{s}\right)-t \int_{|x|>\epsilon}\left(e^{\phi(x)}-1\right) v(d x)\right) .
\end{aligned}
$$


Here $\left(X_{t}^{c}\right)$ is the continuous part of $\left(X_{t}\right)$ and $\eta_{t}$ is such that

$$
\gamma_{t}^{\prime}-\gamma_{t}-\int_{-1}^{1} x\left(v^{\prime}-v\right)(d x)=\sigma_{t}^{2} \eta_{t}
$$

Then we have following theorem of equivalent measures for SVBGAR model.

Theorem 2. Assume that $a=\tilde{a}$. Let

$$
\eta_{s}=\frac{1}{\sigma_{s}^{2}}\left(\tilde{a} \log \left(1-\frac{\rho}{\tilde{\alpha}}\right)-\mu+r-\left(\beta+\frac{1}{2}\right) \sigma_{s}^{2}-\left(\frac{\tilde{a}}{\tilde{\alpha}}\left(1-e^{-\tilde{\alpha}}\right)\right)-\frac{a}{\alpha}\left(1-e^{-\alpha}\right)\right)
$$

and

$$
\begin{aligned}
U_{t}= & \int_{0}^{t} \eta_{s} \sigma_{s} d W_{s}-\frac{1}{2} \int_{0}^{t} \eta_{s}^{2} \sigma_{s}^{2} d s \\
& +\lim _{\epsilon \rightarrow 0}\left(\int_{0}^{t} \int_{\epsilon}^{\infty}(\alpha-\tilde{\alpha}) x J(d x, d s)-t \int_{\epsilon}^{\infty}\left(\frac{\tilde{a} e^{-\tilde{\alpha} x}}{x}-\frac{a e^{-\alpha x}}{x}\right) d x\right),
\end{aligned}
$$

where $J(d x, d s)$ is the jump random measure of $e_{t}$. If we define $\frac{d Q}{d P}=e^{U_{T}}$, then $Q$ is a risk-neutral measure which is equivalent with $P$, so the model is arbitrage free. But the solution of $U_{T}$ is not unique, the model is not complete as other stochastic volatility models.

Proof. If $a=\tilde{a}$, then $\phi(x)=\log \left(\frac{d v^{\prime}}{d v}\right)=(\alpha-\tilde{\alpha}) x$. Since $v(d x)=0$ and $\tilde{v}(d x)=0$ for $x<0$, the condition (32) in Theorem 1 is satisfied as

$$
\int_{-\infty}^{\infty}\left(e^{\phi(x) / 2}-1\right)^{2} v(d x)=a \int_{0}^{\infty} \frac{e^{-\tilde{\alpha} x / 2}-e^{-\alpha x / 2}}{x} d x<\infty,
$$

since $\lim _{x \rightarrow 0} \frac{e^{-\tilde{\alpha} x / 2}-e^{-\alpha x / 2}}{x}=\frac{\alpha-\tilde{\alpha}}{2}<\infty$.

We use the drift $\gamma_{t}^{\prime}=-l(\rho)-\frac{1}{2} \sigma_{s}^{2}$ and $\gamma_{t}=\mu-r+\beta \sigma_{s}^{2}$ and from (35) the $\eta_{s}$ in (36) holds. Since $\sum_{s \leq t,\left|\Delta X_{s}\right|>\epsilon} \phi\left(\Delta X_{s}\right)=\int_{0}^{t} \int_{\epsilon}^{\infty} \phi(x) J(d x, d s)=\int_{0}^{t} \int_{\epsilon}^{\infty}(\alpha-\tilde{\alpha})$ $J(d x, d s)$, (37) is calculated directly from (34).

The difference of measure transformation between BNS and SVBGAR is that the Levy measure of $z_{t}$ in BNS model is finite since it is a compound process but the Levy measure of $e_{t}$ in SVBGAR model is infinite since it is a Gamma subordinator process.

\subsection{European option pricing under SVBGAR model and numerical implementation}

In this section, we assume the model is in the risk-neutral measure. To illustrate the empirical performance of SVBGAR models for derivatives pricing, we 
Table 1. The parameter estimates of SBGAR model from call options.

\begin{tabular}{lcccc}
\hline$a$ & $c$ & $\alpha$ & $\sigma_{0}^{2}$ & $\rho$ \\
\hline 0.0985 & 2.362 & 8.347 & 0.014 & -1.145 \\
\hline
\end{tabular}

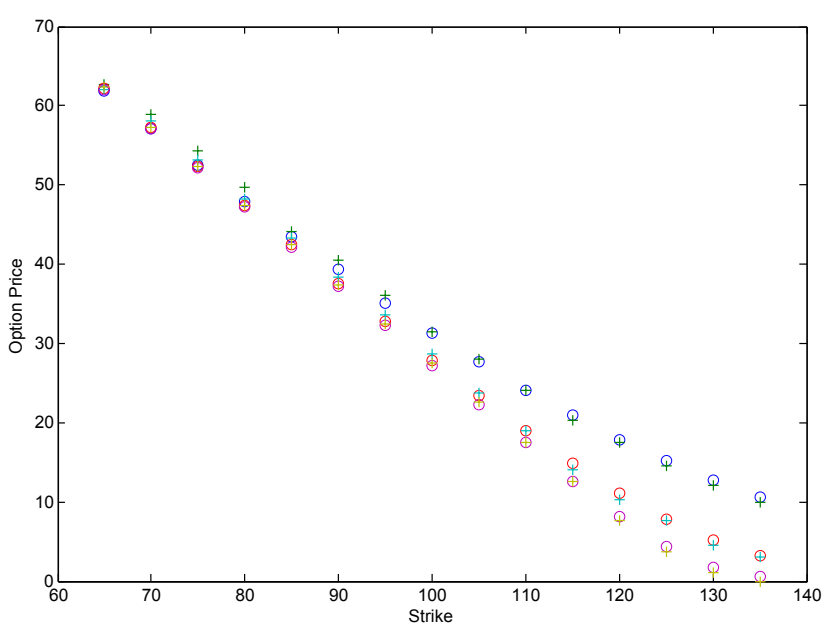

Fig. 1. Actural and predicted prices of the SVBGAR model.

consider a data set of 48 European call options for three different maturities on the IBM stock.

We followed a simple Monto Carlo procedure for constructing the option prices. We calibrated the SVBGAR model by minimizing the mean squared error (MSE) defined as the average squared difference between model-produced option prices and observed prices. We also compute the average absolute error as a percentage of the mean price:

$$
\mathrm{APE}=\frac{1}{\text { mean option price }} \sum_{\text {options }} \frac{\mid \text { market price }- \text { model price } \mid}{\text { number of options }} .
$$

The fitted parameters are given in Table 1 and the observed and fitted option prices are illustrated in Fig. 1. The mean pricing error is $4.485 \%$. The model seems to perform well.

\section{Conclusion}

We started a special Levy process as variance gamma model and BNS stochastic volatility model based on Gamma Ornstein-Uhlenbeck ( $\Gamma$-OU) process. The log 
returns in BNS model with $\Gamma$-OU process have approximately variance gamma distribution as well as volatility clustering effect. We provide a detailed analysis regarding the measure transformations and option pricing of BNS model.

A new SVBGAR model was formulated based on the relationship between gamma and beta variables. The log returns in SVBGAR model still have approximately variance gamma distribution as well as volatility clustering effect. Moreover, the simulation in SVBGAR is much simpler than BNS model. We expect this model can give more efficient way in pricing exotic options by Monte Carlo simulation. As is typical for the more advanced stochas volatility models, the model is arbitrage free but incomplete, which means that there exists more than one equivalent martingale measure. The measure transformation between objective and risk-neutral measures were investigated in this paper.

At last, we researched the performance of SVBGAR model in option pricing. The parameters of SVBGAR model were calibrated from European option prices in market. The model prices were calculated based on the calibration. The comparsion between market and model prices delivered promising performance in option pricing and we hope to provide additional empirical spport in future work.

\section{References}

Barndorff-Nielsen, OE, and N Shephard (2001). Non-Gaussian Ornstein-Uhlenbeck-based models and some of their uses in financial economics, Journal of Royal Statistical Society, 63, 167-241.

Barndorff-Nielsen, OE, E Nicolato and N Shephard (2002). Some recent developments in stochastic volatility modelling, Quantative Finance, 2, 11-23.

Black, F and M Scholes (1973). The pricing of options and corporate liabilities, Journal of Political Economy, 81, 637-654.

Carr, P and D Madan (1998). Option valuation using the fast fourier transform, Journal of Computational Finance, 2, 61-73.

Carr, P, H Geman, D Madan and M Yor (2002). The fine structure of asset returns: An empirical investigation, Journal of Business, 75(2), 305-332.

Carr, P, H Geman, D Madan and M Yor (2003). Stochastic volatility for levy processes, Mathematical Finance, 13, 345-382.

Cont, R and P Tankov (2004). Financial Modelling with Jump Processes. Boca Raton, FL: Chapman and Hall, CRC.

Engle, R (1982). Autoregressive conditional heteroskedasticity with estimates of the variance of U. K. inflation, Econometrica, 50, 987-1008.

Geman, H, D Madan and M Yor (2001). Time changes for levy processes, Mathematical Finance, 11, 79-96.

Geman, H, D Madan and M Yor (2002). Stochastic volatility, jumps and hidden time changes, finance and stochastics, 6, 63-90. 
Heston, S (1993). A closed-form solution for options with stochastic volatility with applications to bond and currency options, Review of Financial Studies 6, 327-343.

Hull, J (2000). Options, futures and other derivative securities, Journal of Finance, 42, 281-300.

Hull, JC and A White (1987). The pricing of options on assets with stochastic volatilities, Journal of Finance, 42, 281-300.

Konikov, M and D Madan (2002). Stochastic volatility via Markov chains, Review of Derivatives Research, 5, 81-115.

Lewis, P, E Mckenzie and D Hugus (1989). Gamma processes, Stochastic Models 5(1), $1-30$.

Madan, D and E Seneta (1990). The variance gamma (VG) model for share market returns, Journal of Business, 63, 511-524.

Madan, D, P Carr and E Chang (1998). The variance gamma process and option pricing, European Finance Review, 2, 79-105.

Merton, RC (1973). Theory of rational option pricing, Bell Journal of Economics and Management, 4, 141-183.

Merton, RC (1976). Option pricing when underlying returns are discontinuous, Journal of Financial Economics, 3, 125-144.

Sato, K (1999). Levy Processes and Infinite Divisibility. Cambridge, UK: Cambridge University Press. 